\title{
Association of G894T polymorphism in endothelial nitric oxide synthase gene with the risk of ischemic stroke: A meta-analysis
}

\author{
MEIYUN WANG, XIUBO JIANG, WENLONG WU and DONGFENG ZHANG \\ Department of Public Health, Medical College of Qingdao University, Qingdao, Shandong 266021, P.R. China
}

Received August 28, 2012; Accepted October 9, 2012

DOI: $10.3892 /$ br. 2012.23

\begin{abstract}
Findings of a previous meta-analysis demonstrated no association between G894T polymorphism in endothelial nitric oxide synthase (eNOS) gene and ischemic stroke. Results of other studies have also been inconsistent. Updated meta-analysis was performed to assess the association between the eNOS gene G894T (rs1799983) polymorphism and the risk of ischemic stroke. Pooled odds ratios (ORs) with $95 \%$ confidence intervals (CIs) from fixed and random effects models were calculated. Heterogeneity in the studies was evaluated using the $\mathrm{I}^{2}$. Meta-regression and the 'leave-one-out' sensitive analysis were used to examine the potential sources of between-study heterogeneity. Publication bias was estimated using the Egger's test. Data were available for 6,607 cases and 6,947 controls from 22 studies. Studies deviating from the Hardy-Weinberg equilibrium (HWE) in the controls and the key contributors to between-study heterogeneity were excluded. Significant associations between eNOS gene G894T polymorphism and the risk of ischemic stroke were observed in the dominant (OR 1.249; 95\% CI, 1.145-1.361), the recessive (OR 1.255; 95\% CI, 1.082-1.456) and the codominant models (OR 1.195; 95\% CI, 1.115-1.281). Moreover, in the subgroup analysis based on the region (Asia and Europe), significant associations were observed between the dominant and codominant genetic models but not in the recessive model. The results of our meta-analysis suggested that eNOS gene G894T polymorphism was associated with the increased risk of ischemic stroke, and that the T allele may be an important risk factor for ischemic stroke. However, further studies are required to confirm this result.
\end{abstract}

Correspondence to: Professor Dongfeng Zhang or Professor Xiubo Jiang, Department of Public Health, Medical College of Qing Dao University, 38 Dengzhou Road, Qingdao, Shandong 266021, P.R. China

E-mail: zhangdf1961@126.com

E-mail: jiangxiubo2005@126.com

Key words: endothelial nitric oxide synthase gene, G894T, gene polymorphism, ischemic stroke, meta-analysis

\section{Introduction}

Ischemic cerebrovascular disease is known to be a multifactorial disorder. Almost $80 \%$ of strokes are ischemic in origin and the remaining cases are hemorrhagic (1). It is the third leading cause of mortality and the most frequent disease leading to disability (2). Genetic factors have recently been found to be highly involved in ischemic stroke (3) through endothelial dysfunction.

Nitric oxide (NO) may also have an anti-thromboembolic effect by reducing platelet adhesion (4) and aggregation (5). NO synthesized produced by the enzyme nitric oxide synthase (NOS) from L-arginine and oxygen is a pluripotent regulatory gas in the cardiovascular system (6). At least three isoforms of NOS have been identified: neuronal (nNOS or NOS-1), inducible (iNOS or NOS-2) and endothelial nitric oxide synthase (eNOS) (eNOS or NOS-3).

eNOS, which produces NO, is most likely to synthesize the $\mathrm{NO}$ that is responsible for maintaining resting cerebral blood flow (7) and its genetic contribution to plasma NO metabolite levels has been demonstrated (8). The involvement of the eNOS gene in the physiology of the vasculature renders it a biologically plausible candidate for study as a susceptibility gene in ischemic stroke (9).

The gene encoding eNOS is located on chromosome 7 (7q35-q36), spanning $21 \mathrm{~kb}$ and comprising 26 exons $(10,11)$. The most relevant eNOS variants are the missense G894T (rs1799983) variant in exon 7 leading to a change of Glu to Asp at site 298 (12). Alterations in this polymorphism may be associated with a reduction in the basal NO production (13).

Studies have been conducted to evaluate the effects of eNOS G894T genetic polymorphism on the risk of ischemic stroke, however, the results were conflicting. In their meta-analysis in 2009, Tao et al (9) reported that a marginal association was observed for homozygosity for the 894T allele with ischemic stroke. Recent studies (14-22) evaluated the correlations between eNOS G894T and the risk of ischemic stroke, however, the results were inconsistent. Therefore, we conducted the present meta-analysis to identify the association between eNOS gene G894T polymorphism and the risk of ischemic stroke.

\section{Materials and methods}

Publication search. The available studies published in English or Chinese (up to July 2012) were identified by extended 
computer-based searches from five databases: i) PubMed; ii) Web of Science; iii) Google Scholar; iv) Wan Fang Med Online and v) China Biology Medical literature database. The search strategy used the following index terms ('eNOS' or 'endothelial nitric oxide synthase' or 'NOS3') and ('genetic' or 'polymorphism' or 'mutation' or 'genes') and ('G894T' or 'Glu298Asp' or 'rs1799983') and ('ischemic stroke' or 'stroke' or 'brain infarction' or 'brain ischemia' or 'cerebrovascular disease'). We also reviewed the references cited in the studies and related studies to identify additional studies not captured in our database searches.

Inclusion criteria. Two investigators carefully reviewed the identified studies independently to determine whether or not an individual study was eligible for inclusion criteria in this meta-analysis. The internationally recognized diagnostic criterion of ischemic stroke was applied (23). The inclusion criteria were: i) case control or cohort study published as an original study to evaluate the association between the G894T (Glu298Asp) polymorphism in eNOS gene and the risk of ischemic stroke; ii) neuroimaging (computed tomography or magnetic resonance imaging) was used to confirm the diagnosis of ischemic stroke; iii) numbers were reported in two comparison groups (ischemic stroke vs. control groups) for genotypes or data provided from which numbers could be calculated and iv) case and control groups in each study had to come from the same ethnicity or period. The most recent and complete studies were chosen if they included overlapping cases/controls.

Exclusion criteria for the studies were: i) the outcomes included patients with hemorrhagic stroke; ii) the genotype frequency was not reported in the study or could not be obtained from authors or iii) subjects were $<18$ years age.

Data extraction. The information from the eligible publications was carefully extracted by two investigators independently, according to the inclusion criteria. First author, journal, year of publication, country, region of the studied population, number of patients in the groups, mean age, male/gender percentage in the case and control groups, and the frequencies of the alleles and the genotype distributions were extracted or calculated for the cases as well as the controls. In case of contradictory evaluations, a third reviewer was used.

Statistical analysis. Departure from Hardy-Weinberg equilibrium (HWE) for the G894T genotype distribution of eNOS gene in the control groups was tested using the Pearson's Chi-square test with exact probability, (HWE; $\mathrm{P}>0.05$ ). Odd ratios (ORs) with $95 \%$ confidence interval (CI) were used to assess the strength of the association between G894T polymorphism in eNOS gene and the risk of ischemic stroke. Polymorphism analysis was performed considering the dominant (TT+GT vs. GG), recessive (TT vs. GT+GG) and codominant (T vs. G) models, respectively. $\mathrm{I}^{2}$ of Higgins and Thompson (24) was used to assess heterogeneity among studies. The DerSimonian and Laird random effect model (REM) was adopted as the pooling method if substantial heterogeneity was present $\left(\mathrm{I}^{2}>50 \%\right)(25)$, otherwise, the fixed effect model (FEM) was selected as the pooling method. Subgroup analyses by region (categorized as European and
Asian) were carried out between the G894T polymorphisms and the risk of ischemic stroke. Meta-regression with restricted maximum likelihood estimation was performed to describe the potentially important covariates, including sample size (the sum of case and control numbers), region (categorized as Asia and Europe), age (ratio of age or median age in the case group to that in the control group) and gender (ratio of male percentage in the case group to that in the control group) that might exert substantial impacts on between-study heterogeneity. If no significant covariates were found to be heterogeneous, the 'leave-one-out' sensitive analysis (26) was carried out to evaluate the key studies with substantial impact on between-study heterogeneity. Influence analysis was conducted (27) to describe whether or not an individual study affected the pooled estimator. Additionally, if the main estimate of an individual study's omitted analysis lay outside the $95 \%$ CI of the combined analysis it was suspected of excessive influence. The modified Egger's test (28) was used to estimate publication bias. The STATA software version 10.0 (Stata Corporation, College Station, TX, USA) was used to carry out statistical analyses. The reported probabilities (P-values) were two-sided. $\mathrm{P}<0.05$ was considered to indicate a statistically significant difference.

\section{Results}

Study characteristics. We found 22 published studies (14-22,29-41) with 23 outcomes (6,607 cases and 6,947 controls) eligible for this meta-analysis connecting G894T polymorphism in eNOS gene to ischemic stroke, including 9 outcomes (29-31,33-37,39), which had made a meta-analysis in 2009 (9). The distribution of genotypes in the control groups were in HWE, with the exception of two studies $(36,39)$. The eligible studies were case/control designs. The general characteristics of the G894T allele and genotype distributions included in this study are shown in Table I.

Meta-analysis results. Pooled analysis was shown in Table II. Unlike a previous meta-analysis (9), this meta-analysis showed a significant association between $\mathrm{T}$ allele and ischemic stroke, in the dominant (REM OR 1.256; 95\% CI, 1.066-1.480); codominant (REM OR 1.237; 95\% CI, 1.084-1.412) and recessive (FEM OR 1.257; 95\% CI, 1.094-1.446) models. A significant association was found between $\mathrm{T}$ allele and an increased risk of stroke following the exclusion of studies deviating from HWE in controls and sensitive analysis for the dominant (FEM OR 1.249; 95\% CI, 1.145-1.361), recessive (FEM OR 1.255; 95\% CI, 1.082-1.456) and codominant (FEM OR 1.195; 95\% CI, 1.115-1.281) models.

A subgroup analysis was also performed by region to evaluate the effect of the eNOS gene G894T polymorphism on the risk of ischemic stroke. Results showed that an increased risk of ischemic stroke associated with the eNOS gene G894T polymorphism was more pronounced in Asia and Europe in the dominant and codominant models but not in the recessive model (Table II and Fig. 1).

With regard to the Asian subgroup, a significant association with the $\mathrm{T}$ allele was detected in the dominant (FEM OR 1.304, 95\% CI, 1.146-1.485) and the codominant (FEM OR $1.326,95 \%$ CI, 1.175-1.496) models, but not in the recessive 
Table I. Characteristics of eNOS gene G894T polymorphism genotype distributions in studies included in this meta-analysis.

Genotype GG/GT/TT

\begin{tabular}{|c|c|c|c|c|c|c|c|c|}
\hline \multirow[b]{2}{*}{ Author } & \multirow[b]{2}{*}{ Year } & \multirow[b]{2}{*}{ Country } & \multirow[b]{2}{*}{ Region } & & \multirow{2}{*}{$\begin{array}{l}\text { Percentage of male } \\
\text { (case/control) }\end{array}$} & \multirow{2}{*}{$\begin{array}{l}\text { P-value for HWE } \\
\text { in control }\end{array}$} & \multirow[b]{2}{*}{ Refs. } \\
\hline & & & & Case & Control & & & \\
\hline Elbaz et al & 2000 & France & Europe & $212 / 187 / 61$ & $163 / 232 / 65$ & $61 / 61$ & 0.24 & 33 \\
\hline Markus et al & 1998 & UK & Europe & $127 / 187 / 47$ & $96 / 104 / 36$ & $58 / 51$ & 0.40 & 37 \\
\hline MacLeod et al & 1999 & Scotland & Europe & 109/125/31 & 154/203/36 & $\mathrm{Na} / 56$ & 0.01 & 36 \\
\hline Szolnoki et al & 2005 & Hungary & Europe & 205/179/23 & $144 / 137 / 14$ & $54 / 53$ & 0.01 & 39 \\
\hline Berger et al & 2007 & Germany & Europe & $869 / 844 / 188$ & $875 / 733 / 138$ & $54 / 60$ & 0.38 & 29 \\
\hline Lv et al & 2004 & China & Asia & $69 / 26 / 5$ & $84 / 14 / 2$ & $62 / 62$ & 0.17 & 35 \\
\hline Cheng et al & 2008 & China & Asia & $235 / 70 / 4$ & $243 / 61 / 5$ & $61 / 61$ & 0.57 & 30 \\
\hline Cui et al & 2007 & China & Asia & $80 / 25 / 9$ & $63 / 12 / 1$ & $61 / 58$ & 0.48 & 31 \\
\hline Hassan et al & 2004 & UK & Europe & $48 / 69 / 19$ & $242 / 294 / 62$ & $68 / 65$ & 0.06 & 34 \\
\hline Saidi et al & 2010 & Tunisia & Africa & 98/170/61 & 229/174/41 & $55 / 55$ & 0.36 & 18 \\
\hline Majumdar et al & 2010 & India & Asia & $124 / 43 / 5$ & $159 / 50 / 5$ & $69 / 67$ & 0.58 & 17 \\
\hline Wang & 2007 & China & Asia & $47 / 9 / 2$ & $49 / 10 / 1$ & $60 / 50$ & 0.46 & 40 \\
\hline Wang & 2007 & China & Asia & $51 / 5 / 2$ & $46 / 13 / 1$ & $59 / 53$ & 1.00 & 40 \\
\hline Zhang et al & 2009 & China & Asia & $107 / 22 / 3$ & $116 / 12 / 0$ & $55 / 55$ & 1.00 & 22 \\
\hline $\mathrm{Xu}$ and Sun & 2004 & China & Asia & $123 / 21 / 4 /$ & $99 / 10 / 0$ & $60 / 83$ & 1.00 & 41 \\
\hline Yan et al & 2011 & China & Asia & $417 / 123 / 5$ & $446 / 102 / 9$ & $65 / 62$ & 0.27 & 20 \\
\hline Guldiken et al & 2008 & Turkey & Asia & $82 / 59 / 5$ & $66 / 61 / 6$ & $51 / 26$ & 0.13 & 14 \\
\hline Luo et al & 2011 & China & Asia & $118 / 29 / 1$ & $115 / 15 / 0$ & $57 / 60$ & 1.00 & 16 \\
\hline Song et al & 2010 & Korea & Asia & $185 / 78 / 2$ & $190 / 40 / 0$ & $46 / 50$ & 0.23 & 19 \\
\hline Yemisci et al & 2009 & Turkey & Asia & $23 / 36 / 8$ & $22 / 47 / 12$ & $63 / 47$ & 0.17 & 21 \\
\hline Li et al & 2011 & China & Asia & $235 / 56 / 9$ & $245 / 53 / 2$ & $64 / 64$ & 1.00 & 15 \\
\hline Di et al & 2004 & China & Asia & $27 / 13 / 0$ & $139 / 30 / 0$ & $\mathrm{Na} / \mathrm{Na}$ & 0.37 & 32 \\
\hline Moe et al & 2008 & Singapore & Asia & $89 / 26 / 3$ & $160 / 42 / 5$ & $76 / 69$ & 0.33 & 38 \\
\hline
\end{tabular}

$\mathrm{Na}$, not available. model (FEM OR 1.273, 95\% CI, 0.848-1.910). No studies deviating from HWE in the Asian subgroup are available. The included studies were in HWE in the controls.

The $\mathrm{T}$ allele was found to be significantly associated with ischemic stroke in the European subgroup (REM OR 1.003; 95\% CI, 0.805-1.249 for the dominant; REM OR 1.026; $95 \%$ CI, 0.893-1.179 for the codominant and FEM OR 1.158; 95\% CI, 0.989-1.357 for the recessive models). Following exclusion of studies $(36,39)$ that deviated from HWE in the controls and the 'leave-one-out' study (33), associations were altered significantly in the dominant (FEM OR 1.206; 95\% CI, 1.074-1.354) and codominant (FEM OR 1.155; 95\% CI, 1.058-1.260) models.

Sources of heterogeneity and sensitive analysis. Before and after excluding the studies deviating from HWE in the controls, strong evidence of heterogeneity was demonstrated in the dominant and codominant inherited models for the studies and the subgroup of Europe (detailed data shown in Table II). However, univariate meta-regression with the covariates of sample size (the sum of case and control numbers), region (categorized as Asia and Europe), age (ratio of mean age in the case group to that in the control group) and gender (ratio of male percentage in the case group to that in the control group) for the above-mentioned polymorphisms, showed that no covariates had a significant impact on between-study heterogeneity (data not shown). The key contributor of the study to between-study heterogeneity was assessed by the 'leave-one-out' sensitive analysis (26) in the included studies and the European subgroup. No significant heterogeneity $\left(\mathrm{I}^{2}<50 \%\right)$ was found in any subgroup following the exclusion of certain studies. However, the association of eNOS gene G894T polymorphism with ischemic risk was significant in the dominant and codominant models in Europe and Asia, but not in the recessive model (detailed data shown in Table II).

Influence analysis. Before and after excluding studies deviating from HWE in the controls as well as the key contributors to between-study heterogeneity, no individual study was found to have excessive influence on the pooled effect in the above-mentioned inherited models. The result of the influence analysis following the exclusion of studies deviating from HWE in the controls is shown in Fig. 2.

Publication bias evaluation. No significant publication bias was detected for ischemic stroke following the exclusion of studies deviating from HWE in the controls and key contributors to 


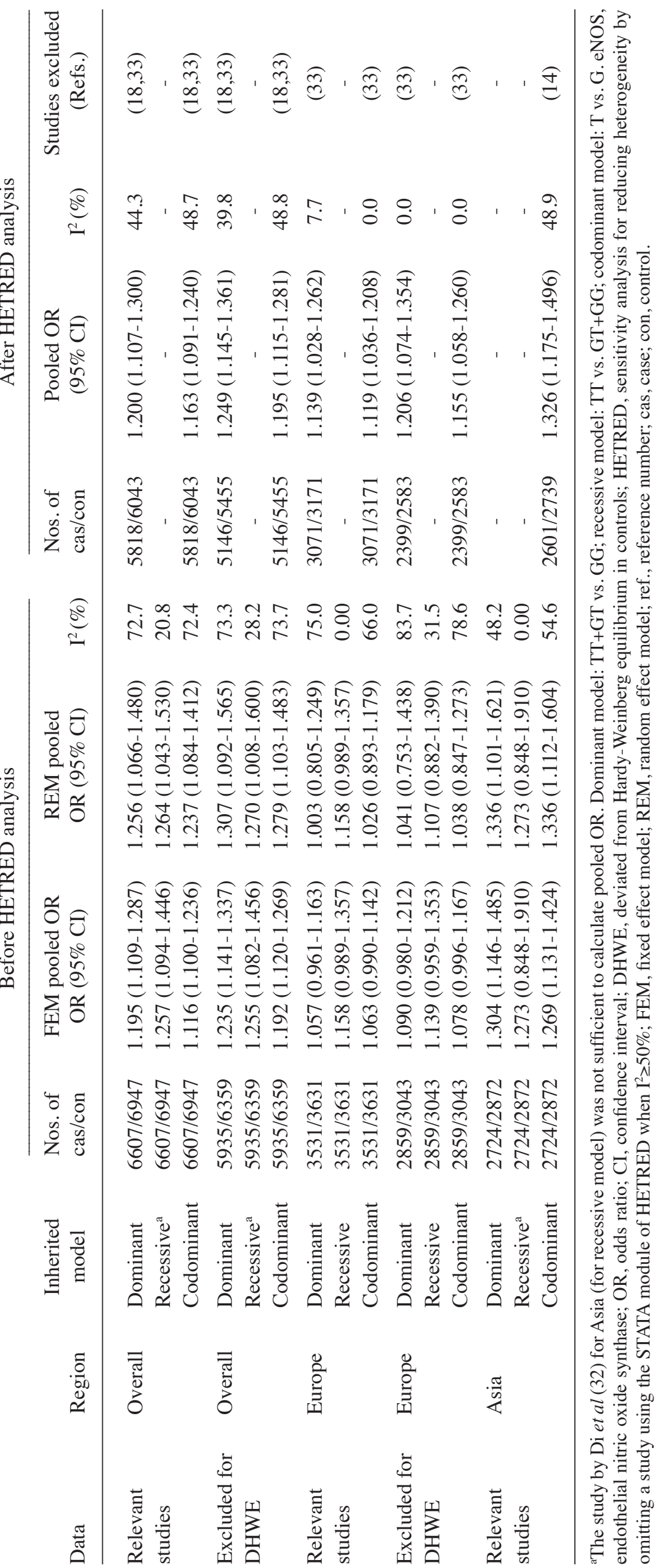




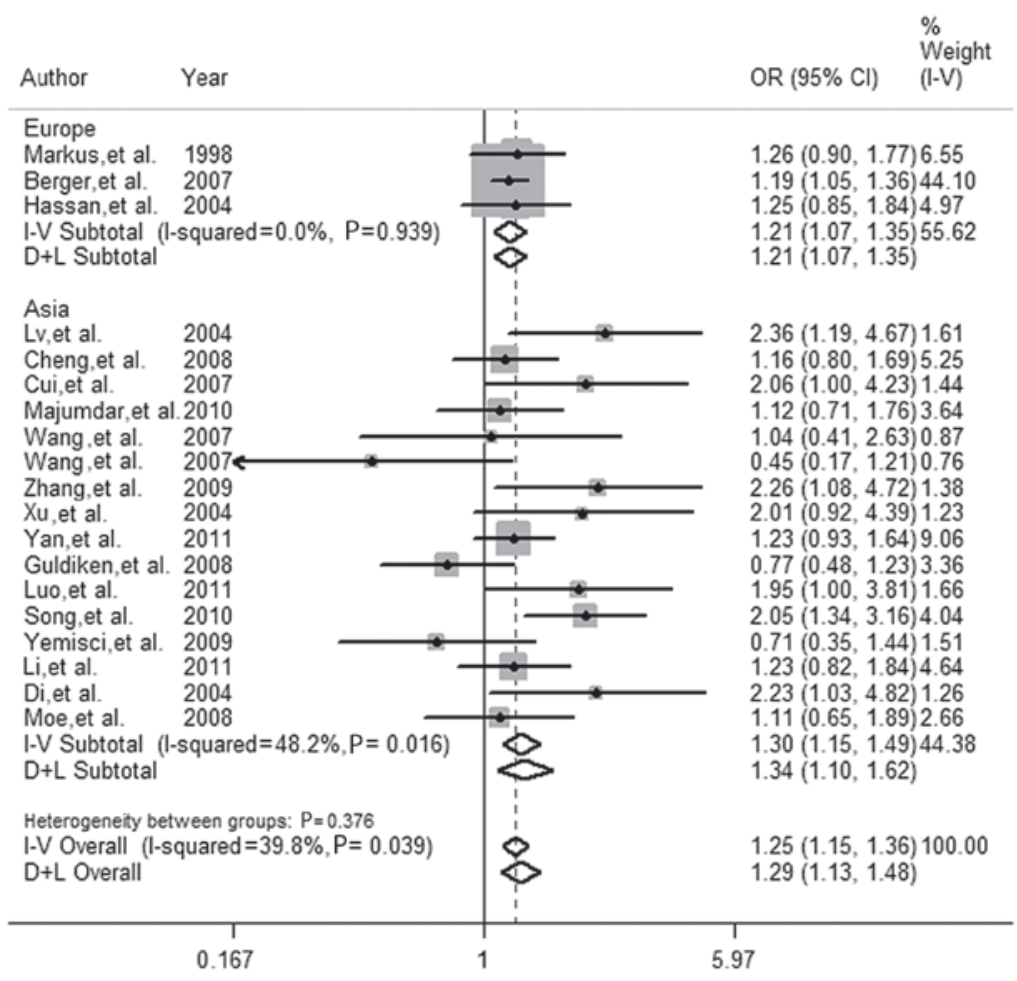

Figure 1. Forest plots of correlation between eNOS gene G894T polymorphism and ischemic stroke risk in dominant (TT+GT vs. GG) model following hte exclusion of studies that deviated from Hardy-Weinberg equilibrium (HWE) in controls, a study in Africa and 'leave-one-out' sensitive analysis. White diamond, the pooled OR. Black squares, the OR in each study, with square sizes that are inversely proportional to the standard error of the OR. Horizontal lines represent $95 \%$ CIs. OR, odds ratio.

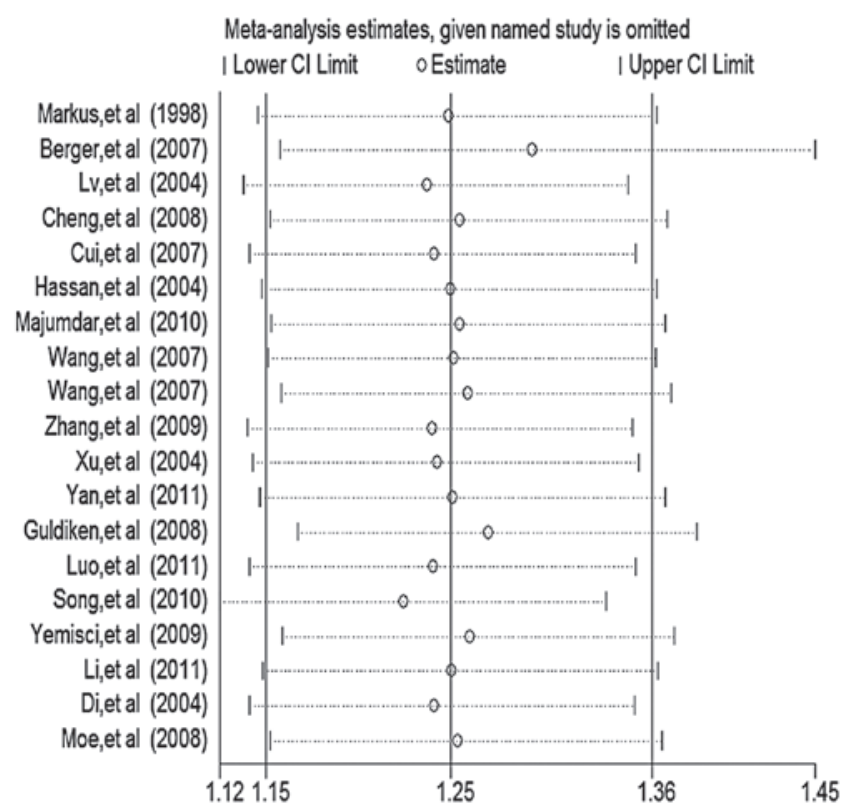

Figure 2. Analysis of influence of meta-analysis estimates, given named study is omitted is shown, i.e., the individual study on the pooled estimate in the dominant model (TT+GT vs. GG) of the eNOS gene G894T polymorphism, following the exclusion of studies deviating from HWE in the controls and 'leave one-out' sensitive analysis. Open circle, the pooled OR, given named study is omitted. Horizontal lines represent the $95 \%$ CIs. OR, odds ratio.

between-study heterogeneity in the above-mentioned three inherited models.

\section{Discussion}

As a pluripotent regulatory gas in the cardiovascular system, $\mathrm{NO}$ is generated by the NOS family of proteins. eNOS-derived $\mathrm{NO}$ is highly involved in the maintenance of vascular homeostasis, including the regulation of the cerebral circulation (9), The mutations at position 298 of the eNOS gene that convert Glu into Asp are considered to be an important risk factor for ischemic stroke.

Recently, studies have been conducted to evaluate the correlation between G894T polymorphism in the eNOS gene and the risk of ischemic stroke. A marginal association was observed for the 894T allele with ischemic stroke (OR, 1.14; 95\% CI, 0.99-1.31) in a meta-analysis conducted by Tao and Chen (9). However, the results obtained remained controversial. Since an individual study has a relatively small number of participants with low power to detect the effect, a meta-analysis may be the appropriate approach to achieve a more definitive conclusion regarding involvement of the eNOS gene G894T polymorphism in the risk of ischemic stroke.

In the present meta-analysis, we retrieved 22 studies $(6,607$ cases and 6,947 controls) to evaluate the association of the eNOS gene G894T polymorphism with ischemic stroke. Of these studies, 13 (2,531 cases and 2,658 controls) comprised Asians (15-17,19,20,22,30-32,35,38,40,41); 8 (3,747 cases and 3,845 controls) Europeans $(14,21,29,33,34,36,37,39)$ and one (18) Africans. The subgroup of Africa was not analyzed due to the limited number of studies available $(n=1)$.

In our meta-analysis, the combined evidence suggested that the eNOS gene G894T polymorphism is associated with 
an increased risk of ischemic stroke, while the results from the subgroup analysis were different in various models.

When comparing the dominant (TT+GT vs. GG) and codominant models (T vs. G), our data indicated that the eNOS gene G894T polymorphism may lead to an increased incidence of ischemic stroke in the Asian as well as the European subgroups, while no significant results were observed for the G894T polymorphism in the recessive model (TT vs. GT+GG) for Asians or Europeans (Table II).

The between-study heterogeneity is common in metaanalysis for genetic association studies (42). Our meta-analysis showed significant between-study heterogeneity in the dominant and codominant models. A number of characteristics, such as sample size, population ethnicity, the ratio of male, the age of cases and deviation from HWE in some studies may be the cause of the between-study heterogeneity. Meta-regression as well as the 'leave-one-out' sensitive analysis were used to reduce the between-study heterogeneity and to explore the potentially important causes of the between-study heterogeneity. No covariate was found to be an important contributor to the between-study heterogeneity by the meta-regression. Following the exclusion of studies that deviated from HWE in the controls $(36,39)$, no substantial variation result was found in the above-mentioned inherited models. The key contributors of the studies to between-study heterogeneity assessed by the 'leave-one-out' sensitive analysis are shown as 'studies excluded' in Table II. After further excluding these studies, low heterogeneity $\left(\mathrm{I}^{2}<50 \%\right)$ was detected in the inherited models and the result was not changed in the relevant studies. However, in the European subgroup, the result was considered significant in the dominant as well as the codominant models. In our meta-analysis, no significant publication bias was observed in the three hereditary models.

In conclusion, our meta-analysis suggested that there was a significant association between the eNOS gene G894T genetic polymorphism and ischemic stroke and the T allele is likely to act as an important ischemic stroke risk factor. The present study is limited by its relatively small sample size and by the fact that the potential biases and confounders in this meta-analysis were not completely precluded. To confirm these findings, additional large-scale studies are required.

\section{Acknowledgements}

This study was supported by the Natural Science Foundation of the Shandong Province (grant no. ZR2010HM100).

\section{References}

1. Goldstein LB, Adams R, Becker K, et al: Primary prevention of ischemic stroke: a statement for healthcare professionals from the Stroke Council of the American Heart Association. Stroke 32: 280-299, 2001.

2. Saver J and Tamburi T: Genetics of cerebrovascular disease. In Neurogenetics. Stefan MP (ed). Oxford University Press, Oxford pp403-411, 2000.

3. Elbaz A and Amarenco P: Genetic susceptibility and ischaemic stroke. Curr Opin Neurol 12: 47-55, 1999.

4. Radomski MW, Palmer RM and Moncada S: Endogenous nitric oxide inhibits human platelet adhesion to vascular endothelium. Lancet 2: 1057-1058, 1987.

5. Radomski MW, Palmer RM and Moncada S: An L-arginine/ nitric oxide pathway present in human platelets regulates aggregation. Proc Natl Acad Sci USA 87: 5193-5197, 1990.
6. Iadecola C, Pelligrino DA, Moskowitz MA and Lassen NA: Nitric oxide synthase inhibition and cerebrovascular regulation. J Cereb Blood Flow Metab 14: 175-192, 1994.

7. Ayata C, Ma J, Meng W, Huang P and Moskowitz MA: L-NAsensitive $\mathrm{rCBF}$ augmentation during vibrissal stimulation in type III nitric oxide synthase mutant mice. J Cereb Blood Flow Metab 16: 539-541, 1996.

8. Wang XL, Mahaney MC, Sim AS, et al: Genetic contribution of the endothelial constitutive nitric oxide synthase gene to plasma nitric oxide levels. Arterioscler Thromb Vasc Biol 17: 3147-3153, 1997.

9. Tao HM and Chen GZ: Endothelial NO synthase gene polymorphisms and risk of ischemic stroke: a meta-analysis. Neurosci Res 64: 311-316, 2009.

10. Marsden PA, Heng HH, Scherer SW, et al: Structure and chromosomal localization of the human constitutive endothelial nitric oxide synthase gene. J Biol Chem 268: 17478-17488, 1993.

11. Nadaud S, Bonnardeaux A, Lathrop M and Soubrier F: Gene structure, polymorphism and mapping of the human endothelial nitric oxide synthase gene. Biochem Biophys Res Commun 198: 1027-1033, 1994.

12. Thomas GD, Zhang W and Victor RG: Nitric oxide deficiency as a cause of clinical hypertension: promising new drug targets for refractory hypertension. JAMA 285: 2055-2057, 2001.

13. Tesauro M, Thompson WC, Rogliani P, Qi L, Chaudhary PP and Moss J: Intracellular processing of endothelial nitric oxide synthase isoforms associated with differences in severity of cardiopulmonary diseases: cleavage of proteins with aspartate vs. glutamate at position 298. Proc Natl Acad Sci USA 97: 2832-2825, 2000.

14. Guldiken B, Sipahi T, Guldiken S, et al: Glu298Asp polymorphism of the endothelial nitric oxide synthase gene in Turkish patients with ischemic stroke. Mol Biol Rep 36: 1539$1543,2009$.

15. Li X, Yang Y, Zhang C and Liu J: The association between endothelial nitric oxide synthase gene polymorphisms and the risk of ischemic stroke in MuDanjiang area. J MuDanjiang Med Univ 32: 31-35, 2011 (In Chinese).

16. Luo N, Li WR, Yan WN, Deng Y, Tan C and Zhang Y: The association of endothelial nitric oxide synthase G894T in exon 7 and the 27-bp repeat in intron 4 genepolymorphisms with cerebral infarction. Med Innov China 8: 1-4, 2011.

17. Majumdar V, Nagaraja D, Karthik N and Christopher R: Association of endothelial nitric oxide synthase gene polymorphisms with early-onset ischemic stroke in South Indians. J Atheroscler Thromb 17: 45-53, 2010.

18. Saidi S, Mallat S, Almawi W and Mahjoub T: Endothelial nitric oxide synthase Glu29 8Asp, 4b/a, and -786T $>$ C gene polymorphisms and the risk of ischemic stroke. Acta Neurol Scand 121: 114-119, 2010.

19. Song J, Kim OJ, Kim H S, et al: Endothelial nitric oxide synthase gene polymorphisms and the risk of silent brain infarction. Int $\mathrm{J}$ Mol Med 25: 819-23, 2010.

20. Yan JT, Zhang L, Xu YJ, Wang XJ, Wang CY and Wang DW: Polymorphisms of genes in nitric oxide-forming pathway associated with ischemic stroke in Chinese Han population. Acta Pharmacol Sin 32: 1357-1363, 2011.

21. Yemisci M, Sinici I, Ozkara H A, et al: Protective role of $27 \mathrm{bp}$ repeat polymorphism in intron 4 of eNOS gene in lacunar infarction. Free Radic Res 43: 272-279, 2009.

22. Zhang ZL, Wei DH, Shen RL and Hu YH: The G894T mutation of the endothelial nitric oxide synthase gene is associated with cerebral infarction in Zhejiang Han nationality. Health Res 29: 180-183, 2009.

23. Hatano S: Experience from a multicentre stroke register: a preliminary report. Bull World Health Organ 54: 541-553, 1976.

24. Higgins JP and Thompson SG: Quantifying heterogeneity in a meta-analysis. Stat Med 21: 1539-1558, 2002.

25. Higgins JP, Thompson SG, Deeks JJ and Altman DG: Measuring inconsistency in meta-analyses. BMJ 327: 557-560, 2003.

26. Patsopoulos NA, Evangelou E and Ioannidis JP: Sensitivity of between-study heterogeneity in meta-analysis: proposed metrics and empirical evaluation. Int J Epidemiol 37: 11481157, 2008.

27. Tobias A: Assessing the influence of a single study in the meta-analysis estimate. Stata Tech Bull 47: 15-17, 1999.

28. Egger M, Davey Smith G, Schneider M and Minder C: Bias in meta-analysis detected by a simple, graphical test. BMJ 315: 629-634, 1997. 
29. Berger K, Stogbauer F, Stoll M, et al: The glu298asp polymorphism in the nitric oxide synthase 3 gene is associated with the risk of ischemic stroke in two large independent case-control studies. Hum Genet 121: 169-178, 2007.

30. Cheng J, Liu J, Li X, et al: Effect of polymorphisms of endothelial nitric oxide synthase on ischemic stroke: a case-control study in a Chinese population. Clin Chim Acta 392: 46-51, 2008

31. Cui JH, Yang G, Su XK, Shao Y and Liu DS: The relationship of angiotensinogen angiotensin-converting enzyme and endothelial nitric oxide synthase gene polymorphisms in predisposition to cerebral infarction. Chin J Gerontol 27: 2197-2199, 2007 (In Chinese).

32. Di Q, Zhan Y, Cheng Y and Ding X: Association of Glu298Asp polymorphism of the endothelial nitric oxide synthase gene with cerebral infarction in aged people. Chin J Gerontol 13: 2004 (In Chinese).

33. Elbaz A, Poirier O, Moulin T, Chedru F, Cambien F and Amarenco P: Association between the Glu298Asp polymorphism in the endothelial constitutive nitric oxide synthase gene and brain infarction. The GENIC Investigators. Stroke 31: 1634-1639, 2000.

34. Hassan A, Gormley K, O'Sullivan M, et al: Endothelial nitric oxide gene haplotypes and risk of cerebral small-vessel disease. Stroke 35: 654-659, 2004.

35. Lv H, Lei T and Zhao L: Correlation between cerebral infarction and nitric oxide synthase gene polymorphism. Chin J Clin Rehabil 8: 4469-4471, 2004 (In Chinese).
36. MacLeod MJ, Dahiyat MT, Cumming A, Meiklejohn D, Shaw D and St. Clair D: No association between Glu/Asp polymorphism of NOS3 gene and ischemic stroke. Neurology 53: 418-420, 1999.

37. Markus HS, Ruigrok Y, Ali N and Powell JF: Endothelial nitric oxide synthase exon 7 polymorphism, ischemic cerebrovascular disease, and carotid atheroma. Stroke 29: 1908-1911, 1998.

38. Moe KT, Woon FP, De Silva DA, et al: Association of acute ischemic stroke with the MTHFR C677T polymorphism but not with NOS3 gene polymorphisms in a Singapore population. Eur J Neurol 15: 1309-1314, 2008

39. Szolnoki Z, Havasi V, Bene J, et al: Endothelial nitric oxide synthase gene interactions and the risk of ischaemic stroke. Acta Neurol Scand 111: 29-33, 2005.

40. Wang L: Study of the polymorphism of eN0S Gene G894T4a/b and ABCAI gene with cerebral infarction in Xinjiang Kazakh Group. Fujian Med Univ, 2007. http://d.g.wanfangdata.com.cn/ Thesis Y1109108.aspx.

41. Xu D and Sun X: Association between the G894T polymorphism in the endothelial constitutive nitric oxide synthase gene and brain infarction. Chin J Gerontol 24: 340-343, 2004 (In Chinese).

42. Munafo MR and Flint J: Meta-analysis of genetic association studies. Trends Genet 20: 439-444, 2004. 